\title{
The State of Pediatric Dental Sedation Literature and Research: A Paucity of Evidence Based Support
}

\begin{abstract}
John E Nathan ${ }^{*}$
${ }^{1}$ Diplomate, American Board of Pediatric Dentistry, Fellow, American Academy of Pediatric Dentistry; Fellow and Master, American Society of Dentistry for Children; Adjunct Professor, Department of Pediatric Dentistry, University of Alabama, Birmingham and Case Western Reserve University, Cleveland; In practice limited to pediatric dentistry, Oak Brook and St. Charles, IL, USA

*Corresponding Author: John E Nathan, DDS, MDS, Diplomate, American Board of Pediatric Dentistry, Fellow, American Academy of Pediatric Dentistry; Fellow and Master, American Society of Dentistry for Children; Adjunct Professor, Department of Pediatric Dentistry, University of Alabama, Birmingham and Case Western Reserve University, Cleveland; In practice limited to pediatric dentistry, Oak Brook and St. Charles, IL, USA; Email: jnathandds@gmail.com; drjohnenathan@gmail.com
\end{abstract}

Received Date: 07-10-2021; Accepted Date: 29-10-2021; Published Date: 05-11-2021

Copyright $^{\odot} 2021$ by Nathan JE, et al. All rights reserved. This is an open access article distributed under the terms of the Creative Commons Attribution License, which permits unrestricted use, distribution and reproduction in any medium, provided the original author and source are credited.

\begin{abstract}
Among the most controversial areas within the field of pediatric dentistry since the latter part of the 20th century has been the utilization of sedative techniques for management of apprehensive and resistive child behaviors when conventional non-pharmacological approaches prove inadequate. Despite a plethora of studies, the vast majority of reports can best be characterized as clinical impression, and retrospective analyses, with a profound paucity of prospective trials representing well-controlled events yielding evidence-based conclusions. Textbook recommendations presented have suggested agent and dosage guidelines, but even more rarely have such been supported by sound research methodologies. Critical analysis of most clinical trials, with few exceptions, are replete with significant shortcomings and design flaws. Some studies including those employing repetitiously flawed methodologies, several in the latter 1980's and early 1990's claim findings which carry little or no validity because of the extent of inherent design deficiencies that confound the ability to draw legitimate conclusions. Despite such, it is not uncommon for techniques and drug dosing recommendations of these works to be in current use. This paper will identify several such studies, concatenated authority which discusses their limited contribution to the literature, and a lack of evidence based support for safety and efficacy. The nature of behavioral clinical

Nathan JE | Volume 2; Issue 3 (2021) | JDHOR-2(3)-035 | Research Article

Citation: Nathan JE, et al. The State of Pediatric Dental Sedation Literature and Research: A Paucity of

Evidence Based Support. J Dental Health Oral Res. 2021;2(3):1-15.


research carries numerous and often insurmountable obstacles in the form of difficult to control variables which will become apparent in the manuscript. A specific aim of this manuscript will be to place in perspective what has been learned from a constructive perspective, where research direction needs to occur, and how to secure evidence based data to permit favorable clinical decisions to be derived regarding agent and dosage selection based on relevant cues to enhance sedation efficacy and patient safety.

\section{Keywords}

Pediatric Sedation; Methodology; Dentistry; Anesthesia

\section{Introduction}

Much remains to be learned about the safe, effective, and predictable use of sedation for managing the manifestations of childhood dental anxiety and resistance. While considerable information has appeared in the pediatric literature as to how to pharmacologically manage challenging child behavior when conventional and mainstream non-pharmacologic approaches fall short or become inappropriate, rarely is substantive data presented to provide empirical evidence based support for what both works and is safe [1,2]. Clinical impression, a few textbook offerings, and largely retrospective analyses comprise the bulk of existing literature dating back to 1960. Rarely are prospective studies presented that include sound research design methodologies. Few exceptions exist that employ non-confounding variables and offer informative insights into current levels of knowledge. Expansion and growth in this area, however, has been encouraging by the recognition that future recommendations for what to include in our pharmacologic arsenals must be supported by well-designed and controlled investigation. In addition, only recently has mention been made of the importance of the development of safety measures to promote and guarantee child well-being by the establishment of national data banks to monitor and record instances of morbidity and mortality when sedative techniques are employed. In the final analysis, it has been long in coming that energies within and outside the field of pediatric dentistry have begun to focus on exploring means and methods to address these deficiencies. Preceding decades have witnessed virtually no interest to look critically at sedation mishaps, their etiology and shortcomings of individual clinician judgment and preparedness [3-5]. Policies of agencies claiming to make true patient advocacy their mission in actuality till now, deferred to litigation and external sources to discipline responsible parties. A more pro-active approach is long overdue and highly welcomed. Only recently has state regulatory agencies, including Illinois for example, begun to initiate requirements for verification of practitioner and office proficiency in management of in-office medical emergencies, and demand mandatory periodic simulation drills. Efforts toward recertification of state licensure permitting sedation, while labor and cost intensive,

Nathan JE | Volume 2; Issue 3 (2021) | JDHOR-2(3)-035 | Research Article

Citation: Nathan JE, et al. The State of Pediatric Dental Sedation Literature and Research: A Paucity of Evidence Based Support. J Dental Health Oral Res. 2021;2(3):1-15. 
have begun to include site-visit inspections where feasible. Funding and personnel demands may be expected to achieve such is likely to be an uphill climb.

Pediatric sedation represents an area for which significant need and improvement in research methodologies exists in which to demonstrate clinical efficacy and safety. Sometimes, obstacles motivated for reasons which contradict illumination present within specialty organizations which impair this pursuit. One such occurrence in this author's opinion, has been a drastic turnaround and lowering of the bar between the pursuits of excellence vs mediocrity. Prior to 2001, the quest for excellence predominated the mission of the American Board of Pediatric Dentistry. Achievement of board certification, occurred for but $15 \%$ of its membership. For reasons related to cost, time and commitment to satisfy this milestone, few pursued its completion. Not unlike other specialty organizations, the American Board of Pediatric dentistry took the position that need existed to drastically increase its membership who demonstrated this level of achievement. To attract increased numbers, a substantial reduction in the demands of the examination process was implemented. To its detriment, emphasis was placed on how to best expand the numbers of Diplomates as quickly as possible at the expense of lowering the bar from the pursuit of excellence to one of minimum of standards. What was once a rigorous and demanding 4 part examination (full day written from an exhaustive reading list, followed by a one hour oral examination a year later, then presentation of 6-7 clinical cases which enabled candidates to demonstrate extensive clinical and diagnostic skills, followed in one year by an all-day site visit with two examiners. Subsequently, the process was reduced to 2 parts, (an abbreviated $1 / 2$ day written exam followed a $1-1 \frac{1}{2}$ oral simulation).

For reasons related to cost and high expectations for candidates to submit and complete extensive examination, only a small percentage sought Diplomate status. It became the perception of the Board of pediatric dentistry and other disciplines that the public essentially expected substantial increases in membership satisfying standards that applied to all health care specialists. Standards for relaxed requirements has become the norm for what represents the most challenging if not potentially dangerous of modalities used in pediatric dentistry. What was once believed to constitute the highest standard, i.e. diplomate status, no longer represents the excellence it once commanded. To the contrary, it seeks to recognize minimum standards to achieve certification. Testing to verify minimum of standards has become the norm. In so doing, sedation experiences within training programs have correspondingly diminished, and utilization of general anesthesia increased out of fear of encountering adverse reactions from the use of higher and more therapeutic dosing [6,7].

Pressures to expand its certified membership exceeded expectations and has currently reached $85 \%$. Doing so came at a cost to drastically lower the bar of knowledge and diminish proficiency in the use of sedative techniques. For some if not many traditionalists, this came as

Nathan JE | Volume 2; Issue 3 (2021) | JDHOR-2(3)-035 | Research Article

Citation: Nathan JE, et al. The State of Pediatric Dental Sedation Literature and Research: A Paucity of Evidence Based Support. J Dental Health Oral Res. 2021;2(3):1-15. 
a step backwards. Diplomate status no longer constituted the "best of the best". For others, it represented opportunity to reward those unwilling to take on the challenges associated with a pursuit of excellence. In any case familiarity with contemporary research literature, particularly in the area of pediatric sedation, was drastically reduced in scientific foundation and clinical application.

As skillful and diverse use of sedation has diminished in both private practice and academic settings as efficacy and safety has lessened, greater use of physical restraint appears to have become accepted practice when practitioner judgment fails to identify effective and safe dosing. A challenge of the contemporary literature is to address such deficiencies and offer clinicians improved choices resulting in enhanced proficiency. Today's challenges are real. Arguments are made that earlier board requirements were excessive that achievement of certification as previously structured were in need of modification for the average specialist. Bottom line appears to have become a standard that declares mediocrity as acceptable. As such, sedation is utilized to a lesser extent and the armamentarium of agents has diminished presumably with a commensurate lack of supporting research. Despite the development of numerous agencies to establish sound guidelines for the elective and safe use of sedation, mishaps continue to be reported [7,8]. Recent sedation deaths of young children have been reported; details regarding conditions, medications used, or efforts to prevent this tragic occurrence are under scrutiny to differentiate idiosyncratic reaction from negligence. Nevertheless, the origin and cause of such outcomes and recurrence of morbidity and mortality can be expected to continue in the absence of sound clinical research [9-11].

The focus of this manuscript will be to identify what we do and do not know, and to discuss what obstacles remain in the pathway to enhancing modalities to best treat the manifestations of interfering and disruptive child behaviors within an office setting. Improvements in the use of sedation for the enhancement of safety, reducing the need for deep sedation and frequent use of unconscious techniques, physical restraint, and adverse outcomes is the target.

\section{Background}

\section{Historical Look at Pediatric Sedation}

Unlike medical practice where invasive procedures are most commonly performed under general anesthesia, expectations in dentistry largely fall under pressures to complete procedures conducted within an office setting on a conscious subject. Fearfulness and a resultant avoidance of care still characterize a percentage of the population. For these subjects, often nonmainstream approaches are needed to provide care. Methods for the provision of care to children lacking cooperative ability for any assortment of reasons suggests a need to exhaust conventional communication strategies before resorting to pharmacological strategies 
(conscious or unconscious) and/or physical restraint. Evidence-based support for nonmainstream techniques that offer both efficacy and patient safety is needed. For some parents, use of general anesthesia is inconceivable; for others, resorting to a less controlled environment to use a sedative technique is equally perceived as dangerous and unacceptable. Deferment of treatment with the hope that their child's interfering behaviors or lack of coping skills will vanish in the near future, replaced by a needed degree of maturation, becomes a logical course of action for parents especially fearful of pharmacologic strategies. For those where time and deferment of care is not possible, the use of physical restraint to permit delivery of care may be the only modality available. The need for well designed research to identify more efficacious pharmacologic strategies become a necessary approach.

For reasons which seem to escape logic pediatric dentists continue to push the limits to which patient safety is protected when attempting to use various sedation agents and dosages in a dental office setting. Such settings in many instances provide a minimum of safety when compared to selecting general anesthetic in a maximally equipped center. Fear of general anesthesia, rational or otherwise, is not uncommon for some parents. Some states have established guidelines that mandate and provide medical coverage for children believed incapable of cooperation. Some identify age restrictions; others neurological constraints. Where age limitations are imposed (Illinois for example, excludes coverage to children under 7 years age if neurologically healthy). In order to receive benefits, children over 6 must show evidence of neurological deficit. Regardless of a lack of cooperative ability, children 7 and older encounter prohibitive costs which no doubt force practitioners and parents to use sedative modalities in a less than maximally equipped facility. Along similar lines, attention directed to the public regarding adverse and catastrophic events associated with misuse of sedative techniques contributes to a reasonable degree of reticence to employ pharmacological modalities in an office setting. Alternatively, the costs associated with a catastrophic event render this justification moot, regardless of the modality or setting. Furthermore, explanation to parents that follow adverse events is daunting and every reasonable effort must be made to avoid such occurrences.

The below review explores existing literature from a historical perspective to present times. While comprehensive and exhaustive review falls outside the scope of this manuscript, focus will remain on aspects of the literature which have contributed to a growing knowledge and understanding of the science of sedative techniques to alleviate patient anxiety and resistance to care. The reader should grasp the complexity of conducting sedation trials which demonstrate the capacity to generate evidence based support for what to use on a daily basis. Critique of methodologies remains easy. Design that secures adequate patient populations, defines and validates assessment of pre-treatment anxiety and uncooperative behaviors, compares agents and dosing used alone and in combination for managing varying levels of apprehension, eliminates confounding variables from interpretation, while maintaining Nathan JE | Volume 2; Issue 3 (2021) | JDHOR-2(3)-035 | Research Article 
desirable levels of consciousness pre-operatively, intra-operatively, and post-operatively is needed.

Lampshire described limitations of single agents and the merit of using combinations of medications he termed "balanced medication" to potentiate the positive aspects of a chosen agent while offsetting any of its downsides [12]. This theoretical assertion serves in a contemporary manner as the basis for many of todays' combinations to best enable drug efficacy and safety avoiding inadvertent induction of deeper planes of depressed consciousness with diminished side-effects. Unfortunately data to substantiate in controlled fashion how to achieve that goal remains lacking. Nevertheless, early studies confirmed the plausible benefits of Lampshire's hypothesis. Robbins study, while limited in subject selection criteria and qualitative or quantitative assessment of anxiety, provided immediate support for the beneficial addition of an anti-emetic to Chloral Hydrate $(\mathrm{CH})$ which produced significantly improved patient behaviors and reduced nausea/emesis when using half the chloral hydrate dosage [13]. The GI upsetting nature of chloral hydrate, the most studied and utilized sedative for visits of long duration of action in children has been explored across numerous retrospective and prospective reports up until and including recent times in efforts to refine its capabilities and application. A perception that appears to have contributed to a decline in $\mathrm{CH}$ popularity and termination of the manufacture of its elixir form has resulted from misuse and abuse of this agent [14]. CH with a capacity to overcome heightened levels of anxiety and resistance in children across long duration visits ( $>20-45$ minutes) has essentially been removed by regulatory agencies because of clinician misuse and abuse, not necessarily scientific evidence. Failure to recognize potentiating disaster by exceeding the toxic dosage of local anesthetic when combined with other sedative agents accounts for sentiment that caused $\mathrm{CH}$ to fall out of favor.

Musselman and McClure hypothesized that varying degrees of apprehension and the relative invasiveness of a planned procedure impacted on agent selection and patient dosing [15]. Within this construct, subjects with mild levels of anxiety warranted low-end dosing, what they termed "preventive medication," in an effort to intercept deterioration in cooperation. Those with heightened levels of anxiety necessitated higher-end dosing, referred to as "management medication." No data was provided to illustrate support for this construct; however, in theory and application, it seems logical that these variables play a significant role in agent and dosage selection that extends to this day. Analysis of research design of much of the contemporary literature, as described below, appears to disregard this premise when designing research methodologies. Studies to date appear to focus on comparing efficacy and safety without regard to varying levels of apprehension and behavioral resistance. Most studies limit their conclusions to comparisons of patient responses to specific dosing, without valid qualification or quantitative incorporation of the specific needs of a given patient. That said, this is by no 
means a shortcoming easily addressed. Absence of inclusion of varying levels of anxiety and uncooperative behavior predominates virtually every study reported in the last two decades.

Prior to the mid-80's, little attention was directed to aspects of agent potency, airway management, and overall patient safety. Textbook references identified $\mathrm{CH}$ dosage guidelines (25 mg/kg sedative dosage and $50 \mathrm{mg} / \mathrm{kg}$ as a hypnotic dosage) for children based on demands for specific patient visits. Manufacturer's recommendations were limited to dosing as a premedication to gain access to the patient but in no way reflected potential to perform invasive treatment. As result of its relative unpredictability and variable responses to varying levels of apprehension, via a textbook suggestion, Trapp offered extended manufacturer recommendations for $\mathrm{CH}$ to $50-70$, and $75 \mathrm{mg} / \mathrm{kg}$ [16]. Until the latter 1980's no hard evidence was presented to suggest or imply that these latter recommendations were warranted or supportable, if not simply dangerous.

Prior to and following 1985, several reports sought to provide evidence of the effectiveness and safety of $\mathrm{CH}$ regimens.

Goodson and Moore reported life threatening occurrences with the use of a potent narcotic, antihistamine, and local anesthetic in a dental context for management of very difficult young children in an office setting [17]. Alphaprodine, adopted from obstetrics for its analgesic characteristics as a potential and rapid-acting modifier of challenging young child behavior, was described for 12 cases, several of which resulted in fatalities associated with profound respiratory depression and cardiovascular collapse. Analysis of the etiology of outcomes included exceeding toxic doses of local anesthetic in addition to excessive narcotic. These findings were instrumental in the removal of the narcotic from clinical use and as per the study reported below of Moore, need emerged for guidelines that defined levels of sedation, patient monitoring and airway management considerations.

Moore compared 60 subjects' (divided into four groups) responses to 20,40 , and $60 \mathrm{mg} / \mathrm{kg}$ dosages of $\mathrm{CH}$ as well as a placebo [18]. He reported mixed results with the lower-end dosing of $\mathrm{CH}$. 4/15 subjects receiving $60 \mathrm{mg} / \mathrm{kg} \mathrm{CH}$ were reported to demonstrate an inability to selfmaintain a patent airway when a head tilt exercise to intentionally and temporarily obstruct the airway was not self-corrected. The result that placebo subjects responded with improved behaviors over $\mathrm{CH}$ subjects might only be explained by the absence of a valid behavioral subject selection criteria which may have included variable apprehension levels ranging from non-anxious to highly anxious subjects in need of a sedative medication. 11/15 subjects successfully self-corrected their airways in this group. The reader was unable to determine if pre-treatment apprehension levels were present to determine if sufficiently anxious subjects were included to warrant the $60 \mathrm{mg} / \mathrm{kg}$ dosing. A conclusion that $60 \mathrm{mg} / \mathrm{kg}$ dosage was too high, or safe, was confounded by the high probability that these subjects were not sufficiently Nathan JE | Volume 2; Issue 3 (2021) | JDHOR-2(3)-035 | Research Article 
anxious from the outset. On a very positive note despite its numerous methodological shortcomings, however, this was among the first studies to draw attention to the need to closely monitor airway patency and relevant physiologic parameters.

In 1985, the AAPD developed its initial round of safety guidelines 19 for the elective use of pediatric sedation in a clinical setting. The guidelines included a need to better define varying levels of sedation, needed parameters for patient monitoring of cardiovascular, respiratory function, and maintenance of protective reflexes. Recommendations included personnel, facility, and resuscitation equipment needs. Similarly, other disciplines including the AAP, ASA, OMFS, to name a few, initiated their own guidelines, some in conjunction with other providers. In any event, updated versions are reported on an ongoing basis. Problematic, however, are issues related to provider compliance following existing guidelines. Regulation and monitoring of this component remains a problem in light of recurrent catastrophic events reported to this day. A finding of Goodson and Moore that cited the effect of exceeding toxic dosages of local anesthetic in combination with sedative agents has continued to this day as one common and underlying cause of catastrophic outcomes.

It became apparent over the next decade that the extent to which sedation success was achieved using recommended agents and dosing often fell short of success. In efforts to avoid mishap by virtue of excessive dosing, this opened the door to the adjunctive use of physical restraint to complete treatment objectives. From this juncture, clinicians felt compelled to find justification of the deployment of physical restraint, (preferably referred to as "protective stabilization") to augment inadequate levels of sedation in younger patients lacking cooperative potential. At this juncture, parents were authoritatively led to believe such measures were appropriate and chosen in an effort to avoid unconscious, more costly, and risky modalities. It became plausible to believe that use of lower, possibly inadequate dosing was a safer alternative to the use of higher dosing which carried potential to induce deeper levels of sedation than were intended. Restraint became a theoretical if not more favorable option. The use of devices that bind children, prevent interfering movement to permit advancement of treatment, however, also carry potential downsides of restriction of chest expansion and respiratory function which must be closely monitored.

Numerous studies during the 1980's and early 1990's made use of a methodology that included binding all child subjects arbitrarily in a restraint device while administering fixed 50\% concentrations of nitrous oxide to its subjects with the objective of comparing hypnotic and higher doses of $\mathrm{CH}$ [20-25]. Measurement of patient movement and crying behaviors was attempted. Behavioral selection criteria was not well defined, nor were qualitative and quantitative analyses of behavioral responses clarified. Interpretations of sedation success by virtue of restraint of patient movement were not addressed, nor was the impact of using a fixed concentration of inhalation analgesia (nitrous oxide-oxygen) described. High incidence of

Nathan JE | Volume 2; Issue 3 (2021) | JDHOR-2(3)-035 | Research Article

Citation: Nathan JE, et al. The State of Pediatric Dental Sedation Literature and Research: A Paucity of Evidence Based Support. J Dental Health Oral Res. 2021;2(3):1-15. 
emesis was reported. Interpretations of drug effects were compromised by not knowing whether nitrous was too high, contributing to nausea, or insufficient to augment the sedative agents tested. Why a fixed $50 \%$ concentration of nitrous oxide was deemed appropriate was not addressed, nor a need to place all subjects in a restraining device explained. Nevertheless this methodology was duplicated in numerous studies; concatenated authority surreptitiously prevailed without substantive data over the next decade while yielding little evidence-based support for agent efficacy, safety, nor appropriateness.

Double and triple blind controlled investigations of Nathan, et al., and Weinstein, et al., respectively, identified behavioral and physiologic responses of young children to varying levels of nitrous-oxide for varying levels of patient anxiety [26,27]. Nitrous oxide was found to be of limited value for moderate to highly apprehensive subjects. Secondly, it was determined that while some subjects benefitted from low levels of nitrous oxide (10-30\%) concentration, others required higher levels before observing any positive effects. While some clinicians advocate high nitrous concentrations to elicit quick initial patient alteration in negative behavior, arguments can be made that provision of such hypoxic dosing is unsafe. That subjects responded in a highly variable manner to differing concentrations negated the merit of using fixed $50 \%$ concentrations, let alone $70-80 \%$ concentrations. Justification for making use of excessive concentrations of nitrous was explained that concentrations could rapidly be returned to safer levels because of its insoluble nature ( uptake or release) in blood . The arbitrary use of fixed 50\% concentrations for all subjects could be expected to confound the opportunity to discern its effect as an adjunctive agent to sedative agents being compared as to therapeutic benefit or detriment. The arbitrary binding of all subjects in a restraining device further confounded interpretations of drug effects when assessing the etiology of patient movement, by virtue of fatigue, anger, or frustration. Nevertheless, persistent use of the Houpt methodology spanned a greater then ten year period yielding less than evidenced based support for its comparisons.

Nathan and West retrospectively compared Chloral hydrate $(\mathrm{CH})$ combinations for both 50 and $70 \mathrm{mg} / \mathrm{kg}$ doses for difficult to manage patients with anti-emetic with and without meperidine, with and without nitrous oxide in a retrospective study of 135 cases [28]. They reported the addition of meperidine to significantly enhance sedations, permit reduced $\mathrm{CH}$ dosage while minimizing or eliminating need for restraint with reduced occurrence of somnolence. When meperidine was included, need for the higher dose of $\mathrm{CH}$ did not improve sedation success and resulted in a high incidence of somnolence and deeper levels of sedation. Hasty, et al., in possibly the most complete and controlled prospective investigation to date, comparing identical regimens, supported the findings of Nathan and West [29]. Well defined selection criteria combined with valid behavioral assessment permitted evidence of the sedation outcomes and patient safety. This regimen of sedative, analgesic, and anti-emetic as result has

Nathan JE | Volume 2; Issue 3 (2021) | JDHOR-2(3)-035 | Research Article

Citation: Nathan JE, et al. The State of Pediatric Dental Sedation Literature and Research: A Paucity of Evidence Based Support. J Dental Health Oral Res. 2021;2(3):1-15. 
essentially served as the gold standard for treating moderate levels of child anxiety when long duration of action is needed till recently. This issue will be raised below.

Renditions of clinician impressions on chloral hydrate use and dosing have appeared. Liu, et al., identified clinical impressions in a summary of sedation for both children and adults [30]. These authors advocated using $50-75 \mathrm{mg} / \mathrm{kg}$ dosing of $\mathrm{CH}$ regardless of age and weight. Suggestions were made, based on the authors' clinical experience, that repeat dosing was appropriate within 30 minutes of initial dosing when such initial dosing proved inadequate. This recommendation, in light of published warnings, essentially ignored manufacturer's maximum single dosage warnings and latent period demands. Recommendations for use of Chloral hydrate in combination with a second sedative, diazepam were also made for both children and adults with claims of safety based on two limited and non-refereed studies involving limited subjects. Similarly, use of midazolam in children was described without reference to significant existing literature. If followed, catastrophic occurrences with endangerment can be expected to occur. Clinical impressions involving both children and adults in these particular opinion papers published in a continuing education journal lacked any resemblance of scientific and evidenced-based support.

Very little has been offered in the pediatric dental sedation literature spanning 2011 to 2021. With one exception, Nathan and Vargas retrospectively compared variable dosing of midazolam with and without meperidine reporting significant improvement with the addition with the addition of meperidine as well as prolonged working time, which offsets a significant shortcoming of midazolam alone [31]. Retrospective comparisons of both $\mathrm{CH}$ with and without meperidine and midazolam with and without meperidine have recently appeared $[32,33]$. Each compared efficacy and safety of varying dosages of these agents for varying levels of anxiety. Sample sizes included comparisons of over 4000 sedations over 35 years of sedation experience. While impressive in numbers, comparisons were not prospective in nature. A simplified and pragmatic delineation of how anxiety levels and what constituted clinical success and safety were described.

\section{Contemporary Controversies Associated With the Use of Devices for the Restriction of Patient Movement}

During this period and extending to the present day, an area of controversy has arisen which focuses on assessment of what constitutes and defines clinical success when using sedation. No general agreement appears to exist amongst clinicians that clarifies the role and justification for needing restraint to accomplish treatment when sedative techniques alone fall short. This is a complex issue. In an absence of evidence-based support for the efficacy of a given sedative technique, (or the use of a restraint device) with patient safety foremost, it is understandable

Nathan JE | Volume 2; Issue 3 (2021) | JDHOR-2(3)-035 | Research Article

Citation: Nathan JE, et al. The State of Pediatric Dental Sedation Literature and Research: A Paucity of Evidence Based Support. J Dental Health Oral Res. 2021;2(3):1-15. 
that error on the side of under-dosage is preferable to inadvertent induction of deeper than desired levels of sedation. Need to complete treatment objectives when sedation proves inadequate leaves three potential scenarios. First, either augment the sedation making use of physical restraint to overcome interfering patient movement; second, increase dosing at the risk of induction of deeper than intended levels of consciousness or depression, or third, abandon sedation for an unconscious technique. In the absence of substantive data to better refine agent and dosage guidelines which clarify predictability, efficacy, and safety, or skilled clinicians with extensive experience in better selecting appropriate dosing, the option of general anesthesia, from a safety perspective, becomes logical, if not clearly attractive. To date, it appears that increasing numbers of clinicians agree that the need for restraints in a persistent manner rules out classifying a sedation as successful. For some however, simply avoiding general anesthesia permits concluding that sedation, while short of optimal, might in a theoretical sense, be considered adequate. Parental attitudes regarding the need for restraint by virtue of inadequate dosing has begun to be appear in the literature [34]. With an expectation that medications will circumvent the need for restraint, majority of parents indicate they find restraint inappropriate, marginal at best, if not certainly undesirable. Postgraduates and residents reporting minimal history of successful experiences with sedation during their training report choosing general anesthesia with a high frequency and an unwillingness to make use of sedation, particularly making use of restricted dosing they were permitted in their training. Those within or having completed advanced training lacking successful sedation experiences fall into this sentiment. Conversely, those with extensive and favorable history of sedation outcomes carry higher expectations for the avoidance of the need for patient restraint and report they anticipate making greater use of sedative pathways [35].

Surveying sedation practices of advanced training programs with respect to agents and dosing, Wilson and Nathan reported in an effort to minimize or eliminate serious risk, the vast majority of programs reported reduced both their arsenal of agents/combinations used for sedation as well as dosing ranges they considered safe [9]. Midazolam was reported as the single agent most selected for pediatric patients. Limitations in agent selection appears related to the desirability to make use only of agents with a capacity for reversibility. As such, only benzodiazepines and narcotics possess that capacity. As result, agents such as chloral hydrate, diazepam, ketamine have fallen out of favor. Dosages of midazolam ranged from $0.3-0.5 \mathrm{mg} / \mathrm{kg}$ in an effort to avoid mishap. Conversely, data to support the efficacy of this dosage range, however, is lacking with respect to control of anything other than ultra-short and short duration of action and minimal anxiety levels. Need for restraint for more moderate levels of anxiety or procedures of longer duration appears the rule rather than exception. Data from other retrospective studies 34-35 which have retrospectively compared wide dosage ranges of 0.5$1.5 \mathrm{mg} / \mathrm{kg}$ for midazolam alone, or in combination, with and without oral meperidine report significant improvement in efficacy, longer duration of action, and minimal or no need for restraint. Experience and confidence levels with higher dosing levels appears lacking. Because

Nathan JE | Volume 2; Issue 3 (2021) | JDHOR-2(3)-035 | Research Article

Citation: Nathan JE, et al. The State of Pediatric Dental Sedation Literature and Research: A Paucity of Evidence Based Support. J Dental Health Oral Res. 2021;2(3):1-15. 
parental perspectives, however, now appear to lean in the direction of not believing in the value or merit of restraint, clinician preference for using restraint appears waning.

Accreditation standards for advanced training programs appears to be evolving. Programs electing to reduce exposure and experience in the use of a broad spectrum of sedative techniques are under scrutiny to expand their curricula and hands-on sedation experience. Program director and faculty expertise, experience, and comfort levels can be expected to govern the extent to which accreditation norms can be established. State regulation and institutional constraints remain variable. Programs making frequent use of general anesthesia, from an institutional perspective, are internally reviewed favorably due to monetary gains from the cost of care generated. Similarly, deferral of in-clinic risk to anesthesiologists has merit and responsibility from the perspective of avoidance of adverse reactions/outcomes. Whether or not the best interests of patients and parents are best served by the use of general anesthesia when treatment needs are limited and apprehension levels moderate becomes a matter for accreditation of teaching programs to determine. The extent to which an obligation exists for exposure of postgraduate students and residents to a diverse repertoire of agents, routes of administration and available modalities will determine future curriculum directions.

Studies from 2011 to the present underlie a continued research need. Unkel, et al., Weerink, et al., McMorrow and Abromo and Mohite, et al., described the potential use and benefit of dexmedetomidine for pediatric dental patients [36-39]. Conduct of prospective pediatric dental sedation studies are lacking during this past decade. One study recently examined the safety associated with the use of dexmedetomidine in combination with hydroxyzine and $50-70 \%$ nitrous oxide. No data or analysis with respect to efficacy was discussed or revealed. Belief that conclusions of safety accompanied this regimen remain somewhat questionable in light of an absence of data that explored efficacy and that $35 \%$ of subjects experienced intra- or postoperative hypotension. Interest in future study of this agent, administered orally, parenerally, and intranasally appears high.

Mittal, et al., explored the pediatric sedation literature with respect to the use of this agent [40]. Conclusions paralleled previous reports and this report as to the lack of evidenced based supporting data that demonstrates efficacy and safety for this and other pediatric sedation regimens. Making use of a Cochrane review the authors described several sources of bias which included improper allocation, use of blind conditions, incomplete outcome data, poor selection criteria for agent and dosing and questionable sedation assessment. This paper offered considerable insight as to how future studies might be conducted to eliminate bias from a perspective that focuses on improved basis for patient selection, sample sizes, use of validated sedation scoring tools. 


\section{Conclusion and Prerequisites for Future Study}

Without well designed and controlled investigations, reports of mishaps and adverse reactions, some with catastrophic outcomes can be expected to continue to this day. Contemporary removal of agents from sedation arsenals and pharmacy formularies, by virtue of institutional and state regulation, have reduced or limited the use of sedation for management of moderate to severe apprehension predominates clinician and resident use of sedation techniques. Improved research methodologies to provide evidenced based support are needed. While surveys of practitioner impressions are useful to some degree, data that provides valid documentation of specific patient characteristics, subject selection criteria must serve as the first component of a controlled study.

1. Patient selection on the basis of previous experience having been negative, and/or valid screening measures to categorize anxiety levels and behavioral resistance must be incorporated

2. Non-confounding agents or combinations must be compared. Agents at varying dosages should be compared with one another under blind conditions

3. If nitrous oxide effects are explored, control groups must be included that look at varying concentrations of nitrous as well as a no nitrous condition

4. Definitive and reliable qualitative and quantification of anxiety and behavioral resistance must be corroborated for experimental conditions

5. Assessments of behavioral and anxiety ratings need be valid and reproduce-able

6. Physiological monitoring, where appropriate should reflect pre-treatment baselines, specific intra-op intervals, immediate post-treatment, and recovery parameters

7. Recording of aberrations in vital signs, protective reflexes should occur

8. Retrospective studies retain their usefulness when subject numbers are of sufficient magnitude that patient differences can be readily ascertained

9. The methodology of future designed behavioral studies should however, be prospective in nature, wherever possible

\section{Conflict of Interest}

It is stated that there are no conflicts of interest between the proponents and participants in the present work.

\section{References}

1. Nathan JE. The evolution and changing patterns of behavioral management of challenging childhood dental anxiety: A crossroads. Dentistry. 2016;6(5):1-13. 
2. Nathan JE. Contemporary pharmacologic management of the difficult pediatric dental patient: Scientific foundations for efficacy and safety. Alpha Omegan. 2007;99(2):78-82.

3. Wilson S. A review of important elements in sedation study methodology. Pediatric Dentistry. 1995;17:40612.

4. Nathan JE. Pediatric sedation: predictable and practical approaches to avoid mishaps, adverse reactions, and catastrophic outcomes, J Pharmacol and Clin Res. 2017;2(4):1-3.

5. Nathan JE. What will it take to prevent another pediatric sedation or anesthesia fatality? Dentistry. 2017;7(7):1000.

6. Nathan JE. Horrifics of pediatric sedation: when sedation guidelines are disregarded. Surg. 2017;5(1):1-4.

7. Nathan JE. Board certification in pediatric dentistry: once it represented pursuit of excellence, Interventions. Ped Dent J. 2019;2(5):170-2.

8. Nathan JE. Evolution of board certification in pediatric dentistry: changing patterns between enhancing quality vs expanding numbers of qualifying members and its implications for future training. J Oral Health and Dentistry. 2018;2(6):653-5.

9. Wilson S, Nathan JE. A survey study of sedation training in advanced pediatric dentistry programs: thoughts of program directors and students. Pediat Dent. 2011;33(4):352-9.

10. Coté CJ, Karl HW, Notterman DA, Weinberg JA, McCloskey C. Adverse sedation events in pediatrics: analysis of medications used for sedation. Pediat. 2000;106(4):633-44.

11. Cravero JP, Blike GT, Beach M, Gallagher SM, Hertzog JH, Havidich JE, et al. Incidence and nature of adverse events during pediatric sedation/anesthesia for procedures outside the operating room: report from the pediatric sedation research consortium. Pediat. 2006;118(3):1087-96.

12. Lampshire LE. Balanced medication. J Dent Child. 1959;26:25-31.

13. Robbins MB. Chloral hydrate and promethazine as premedicants for the apprehensive child. J Dent Children. 1967;34(5):327.

14. Nathan JE. The disappearance of chloral hydrate as an effective sedation for management of difficult young pediatric dental patients: an inappropriate and unfortunate outcome. J Pharmacol Clin Res. 2018;6(1):1-3.

15. Musselman RJ, McClure DB, Kopel HM. Pharmacotherapeutic approaches to behavior management. Behavior management in dentistry for children. Philadelphia: WB Saunders Co. 1975:146-55.

16. Trapp LD. Sedation of children for dental treatment Dr. Trapp. 1982.

17. Goodson JM, Moore PA. Life-threatening reactions after pedodontic sedation: an assessment of narcotic, local anesthetic, and antiemetic drug interaction. JAMA. 1983;107(2):239-45.

18. Moore PA, Mickey EA, Hargreaves JA, Needleman HL. Sedation in pediatric dentistry: a practical assessment procedure. JADA. 1984;109(4):564-9.

19. Reference manual elective use of sedation and general anesthesia in pediatric dentistry. Pediatric Dentistry. 1985.

20. Houpt MI. Comparisons of Chloral Hydrate dosiong for young pediatric dental patients. Dent. 1985;52: 3649.

21. Houpt MI, Weiss NJ, Koenigsberg SR. Comparisons of chloral hydrate with and without nitrous oxide in children. JDC.

22. Houpt, MI. Comparioson of $\mathrm{CH}$ with and without promethazine IKN the sedation of children. J Den t Child. 1985;7(1):41-6.

23. Poorman TL, Farrington FH, Mourino AP. Comparison of a chloral hydrate/hydroxyzine combination with and without meperidine in the sedation of pediatric dental patients. Pediatr Dent. 1990;12(5):288-91.

24. Needleman HL, Joshi A, Griffith DG. Conscious sedation of pediatric dental patients using chloral hydrate, hydroxyzine and nitrous oxide-a retrospective study of 382 sedations. Pediatr Dent. 1995;17:424-31.

25. Chowdhury J, Vargas KG. Comparison of chloral hydrate, meperidine, and hydroxyzine to midazolam regimens for oral sedation of pediatric dental patients. Pediatr Dent. 2005;27(3):191-7.

Nathan JE | Volume 2; Issue 3 (2021) | JDHOR-2(3)-035 | Research Article 
26. Weinstein P, Domoto PK, Holleman E. The use of nitrous oxide in the treatment of children: results of a controlled study. JADA. 1986;112(3):325-31.

27. Nathan JE, Venham LL, West MS, Werboff J. The effects of nitrous oxide on anxious young pediatric patients across sequential visits: a double-blind study. ASDC J Dent Children. 1988;55(3):220-30.

28. Nathan JE, West MS. Comparison of chloral hydrate-hydroxyzine with and without meperidine for management of the difficult pediatric dental patient. JDC. 1987;54(6):437-44.

29. Hasty MF, Vann WF, Dilley DC, Anderson JA. Conscious sedation of pediatric dental patients: an investigation of chloral hydrate, hydroxyzine pamoate, and meperidine vs. chloral hydrate and hydroxyzine pamoate. Pediatr Dent. 1991;13(1):10-18.

30. Liu Z. Sedation for children and adults using chloral hydrate and other agents. Compendium: Excelsior. 2006;7(12).

31. Nathan JE, Vargas KG. Oral midazolam with and without meperidine for management of the difficult young pediatric dental patient: a retrospective study. Pediatr Dent. 2002;24(2):129-38.

32. Nathan JE. Controversies in behavior guidance and management of challenging pediatric dental behaviors and anxiety: avoidance of restraints wherever possible. J Pediatr Dis Neonatal Care. 2018;301-5.

33. Nathan JE. Retrospective comparisons of varying dosages of chloral hydrate with and without meperidine for varying levels of anxiety: 35 years of Sedation experience. J Clin Pediat Dent. 2021.

34. Nathan JE. Comparisons of variable dosages of Midazolam with and without meperidine for varying levels of anxiety: Data accumulated across 35 years Clinical experience. Fall Winter. 2021.

35. Wilson S, Houpt M. Project USAP 2010: use of sedative agents in pediatric dentistry-a 25-year follow-up survey. Pediat Dent. 2016;38(2):127-33.

36. Unkel JH. A retrospective evaluation of the safety profile of dexmedetomidine for pediatric dental sedation. Ped Dent. 2021;41(2)129-32.

37. Weerink MA, Struys MM, Hannivoort LN, Barends CR, Absalom AR, Colin P. Clinical pharmacokinetics and pharmacodynamics of dexmedetomidine. Clin Pharmacokinetics. 2017;56(8):893-913.

38. McMorrow SP, Abramo TJ. Dexmedetomidine sedation: uses in pediatric procedural sedation outside the operating room. Pediat Emerg Care. 2012;28(3):292-6.

39. Mohite V, Baliga S, Thosar N, Rathi N. Role of dexmedetomidine in pediatric dental sedation. J Dent Anesth Pain Med. 2019;19(2):83-90.

40. Mittal N, Goyal A, Jain K, Gauba K. Pediatric dental sedation research: Where do we stand today? J Clinical Ped Dent. 2015;39(3):284-91. 